\title{
On black hole masses, radio-loudness and bulge luminosities of Seyfert galaxies
}

\author{
Xue-Bing $\mathrm{Wu}^{1,2}$ and J. L. $\mathrm{Han}^{2,3}$ \\ ${ }^{1}$ Department of Astronomy, Peking University, Beijing 100871, PR China \\ ${ }^{2}$ National Astronomical Observatories, Chinese Academy of Sciences, Beijing 100012, PR China \\ ${ }^{3}$ The partner group of Max-Planck-Institut für Radioastronomie at National Astronomical Observatories of China \\ e-mail: hjl@bao.ac.cn
}

Received 8 June 2001 / Accepted 19 September 2001

\begin{abstract}
We estimated black hole masses for 9 Seyfert 1 and 13 Seyfert 2 galaxies in the Palomar and CfA bright Seyfert samples using the tight correlation between black hole mass and bulge velocity dispersion. Combining other 13 Seyfert 1s and 2 Seyfert $2 \mathrm{~s}$ in these samples but with black hole masses measured recently by reverberation mapping and stellar/gas dynamics, we studied the correlations of black hole masses with radio-loudness and bulge luminosities for a sample of 37 Seyfert galaxies. We found that if radio-loudness is measured using the optical and radio luminosities of the nuclear components, the black hole masses of radio-loud Seyfert 1s tend to increase with the radio-loudness. The black hole masses of all Seyfert galaxies increase with the radio power, but Seyfert galaxies have larger radio powers than nearby galaxies with the same black hole masses. In addition, the correlation between black hole masses and bulge $V$-band luminosities for Seyfert galaxies is consistent with that found for quasars and normal galaxies. The combined sample of 37 Seyfert galaxies, 15 quasars and 30 normal galaxies suggests a possible universal nonlinear relation between black hole and bulge masses, $M_{\mathrm{BH}} \propto M_{\text {bulge }}^{1.74 \pm 0.14}$, which is slightly steeper than that found recently by Laor (2001) for a smaller sample. This nonlinear relation is supported by a larger sample including 65 Seyfert galaxies. The different $M_{\mathrm{BH}} / M_{\text {bulge }}$ ratio for galaxies with different bulge luminosities or different black hole masses may be explained by this relation. These results are consistent with some theoretical implications and are important for understanding the nature of radio emissions and the formation and evolution of supermassive black holes and galaxies.
\end{abstract}

Key words. black hole physics - galaxies: active - galaxies: nuclei - galaxies: Seyfert

\section{Introduction}

Supermassive black holes (SMBHs), with masses in the range of $10^{6}$ to $10^{9} M_{\odot}$, have been suggested to exist in the center of quasars and active galactic nuclei (AGNs). Accretion onto these SMBHs may account for the huge power of these energetic objects (Lynden-Bell 1969; Rees 1984). Recently, the masses of central objects in 20 Seyfert galaxies and 17 nearby quasars have been measured with the reverberation mapping technique (Ho 1999; Wandel et al. 1999; Kaspi et al. 2000), which confirmed the existence of SMBHs in the center of these objects. On the other hand, a lot of observations using gas and stellar dynamics indicated that SMBHs probably also exist in the center of our Galaxy (Ghez et al. 1998; Genzel et al. 1997) and in the nuclei of many normal galaxies (Kormendy \& Richstone 1995; Magorrian et al. 1998; Kormendy \& Gebhardt 2001). However, the apparent inactive feature of normal galaxies seems to suggest that the central engines of these galaxies may be different from those in quasars

Send offprint requests to: Xue-Bing Wu, e-mail: wuxb@bac.pku.edu.cn and AGNs. It was suspected that advection-dominated accretion flows (ADAFs; see Narayan et al. 1998; Kato et al. 1998 for reviews) with very low accretion rate and low radiative efficiency may exist in the nuclei of normal galaxies (Fabian \& Rees 1995; Di Matteo \& Fabian 1996), while quasars and most AGNs probably host the standard geometrically thin accretion disks (Shakura \& Sunyaev 1973; Novikov \& Throne 1973) with higher accretion rate.

One interesting result found recently in the searches of SMBHs in nearby galaxies is the correlation of black hole masses with the properties of galactic bulges. Although with large scatters, the black hole masses seem to correlate with bulge luminosities (Kormendy \& Richstone 1995; Magorrian et al. 1998). This also leads to the finding that the black hole mass, $M_{\mathrm{BH}}$, is possibly proportional to the bulge mass, $M_{\text {bulge, }}$, though the mass ratio found by different authors was different, in the range of $0.2 \%$ to $0.6 \%$ (Kormendy \& Richstone 1995; Magorrian et al. 1998; Ho 1999). Laor $(1998,2001)$ recently found that some nearby quasars and Seyfert galaxies follow nearly the same $M_{\mathrm{BH}^{-}}$ bulge luminosity relation as normal galaxies, and suggested a universal nonlinear relation, $M_{\mathrm{BH}} \propto M_{\text {bulge }}^{1.54 \pm 0.15}$, for both normal and active galaxies. This means that the 
$M_{\mathrm{BH}} / M_{\text {bulge }}$ ratio is not constant for galaxies with different bulge luminosities. Recently, a significantly tight correlation of $M_{\mathrm{BH}}$ with the bulge velocity dispersion $\sigma$ was also found for nearby galaxies (Gebhardt et al. 2000a; Ferrarese \& Merritt 2000). More recent studies indicated that 11 Seyfert galaxies with $M_{\mathrm{BH}}$ measured by reverberation mapping follow the same $M_{\mathrm{BH}}-\sigma$ relation as for normal galaxies (Gebhardt et al. 2000b; Ferrarese et al. 2001), implying another possible universal relation for both normal and active galaxies. The correlations of the SMBH mass with the properties of the galactic bulge strongly suggest a tight connection between the formation and evolution of the SMBH and galactic bulge, though the nature of this connection is still in debate (Haehnelt \& Rees 1993; Haiman \& Loeb 1998; Silk \& Rees 1998; Haehnelt et al. 1998; Kauffmann \& Haehnelt 2000; Adams et al. 2001).

Nonthermal radio emissions of quasars and AGNs are believed to be probably produced by relativistic electrons that are powered by jets (Begelman et al. 1984; Blundell \& Beasley 1998). Similar radio emissions have been detected in the nuclei of normal elliptical galaxies (Sadler et al. 1989) and also in some spiral galaxies (Sadler et al. 1995). Recently, some studies have indicated that the radio power may be directly correlated with the black hole mass. Franceschini et al. (1998) found a very tight relation between black hole mass and radio power in a small sample of nearby mostly non-active galaxies. McLure et al. (1999) estimated the black hole masses for a sample of AGNs using $M_{\mathrm{BH}}$-bulge mass relation found by Magorrian et al. (1998) and noted they follow the same correlation with radio power as found by Franceschini et al. (1998). However, Laor (2000) recently argued that the $M_{\mathrm{BH}}$ and radio power of a sample of $87 z<0.5$ Palomar-Green (PG) quasars (Schmidt \& Green 1983; Boroson \& Green 1992) do not follow the tight correlation suggested by Franceschini et al. (1998), because quasars usually have over 100 times larger radio power than normal galaxies at a given $M_{\mathrm{BH}}$. He suggested that the larger scatters of radio power at a given $M_{\mathrm{BH}}$ may be simply due to the different levels of overall continuum luminosity of different objects. Laor (2000) also noted that the radio-loud quasars seem to host more massive black holes than radioquiet quasars. Very recently, Ho \& Peng (2001) studied the radio-loudness of bright Seyfert 1 galaxies using the nuclear radio and optical luminosities, and suggested that the majority of Seyfert 1 nuclei in their sample are essentially radio loud. Therefore, it is useful and feasible to check if these radio-loud Seyfert nuclei host more massive black holes than radio-quiet ones and if the Seyfert galaxies still follow the same correlation between the radio power and black hole masses of nearby galaxies and quasars (Franceschini et al. 1998; Laor 2000). In this paper we will try to derive the SMBH masses for a number of Seyfert galaxies and study their correlations with radio power and bulge luminosities. Further studies of these correlations will probably provide some important clues to help us understand the nature of radio emissions in active

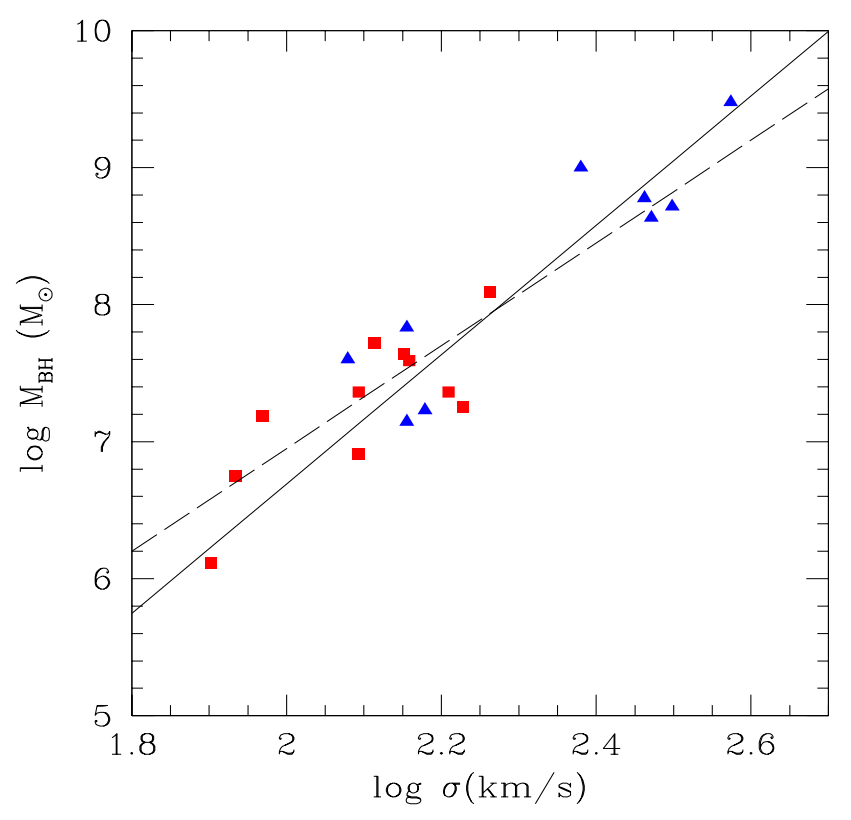

Fig. 1. The black hole mass against the velocity dispersion for Seyfert galaxies. Squares and triangles represent the SMBH masses measured by reverberation mapping and stellar/gas dynamics, respectively. The solid and dashed lines correspond to the correlation found by Merritt \& Ferrarese (2000) and Gebhardt et al. (2000a), respectively.

and normal galaxies and the formation and evolution of SMBHs and galaxies.

\section{Estimating the SMBH masses of Seyfert galaxies}

Currently the SMBH masses of a few weak AGNs have been well measured by stellar dynamics, ionized gas dynamics and water maser dynamics (for a summary see Table 1s in Ho 1999; Gebhardt et al. 2000a). Using the reverberation mapping technique, the SMBH masses of 20 Seyfert galaxies and 17 bright quasars have been recently estimated (Ho 1999; Wandel et al. 1999; Kaspi et al. 2000). However, for most Seyfert galaxies, it is difficult to measure the SMBH mass using these methods because of either the large nuclear luminosity or the lack of long-term variability monitoring and precise measurements of characteristic velocity dispersions in the broad emission line region.

Since the tight correlation between the SMBH mass and bulge velocity dispersion was found for both normal and active galaxies (Gebhardt et al. 2000b; Ferrarese et al. 2001), it may be straightforward to estimate the SMBH mass from the measured bulge velocity dispersion. The correlation between the black hole mass and nuclear velocity dispersion for Seyfert galaxies with both measured nuclear velocity dispersions and black hole masses is demonstrated in Fig. 1. The SMBH masses of 11 Seyfert galaxies (squares in Fig. 1) were estimated by reverberation mapping (Wandel et al. 1999) and 9 (triangles in Fig. 1) by the dynamical method (Ho 1999; Gebhardt et al. 2000a). The values of their central velocity dispersions were taken 
from Nelson \& Whittle (1995) and Ferrarese et al. (2001). Figure 1 clearly shows that Seyfert galaxies follow the same $M_{\mathrm{BH}}-\sigma$ relation as normal galaxies (Gebhardt et al. 2000a; Merritt \& Ferrarese 2000). Although measurements of the central velocity dispersions of these Seyfert galaxies were made by different groups, the small scattering around the $M_{\mathrm{BH}}-\sigma$ relation indicates that these measurements were reliable and the systematic errors may not be important (Ferrarese et al. 2001). In this paper, we adopt the $M_{\mathrm{BH}}-\sigma$ relation found by Merritt \& Ferrarese (2001), namely,

$M_{\mathrm{BH}}=1.3 \times 10^{8} M_{\odot}\left(\sigma / 200 \mathrm{~km} \mathrm{~s}^{-1}\right)^{4.72}$,

to derive the SMBH masses for Seyfert galaxies with measured nuclear velocity dispersions. Using a slightly flatter relation found by Gebhardt et al. (2000a) does not cause significant changes in our results.

In the next sections we will compare some properties of Seyfert galaxies with those of quasars. Only a few quasars have $\mathrm{SMBH}$ masses determined by reverberation mapping. Laor (1998) adopted an empirical relation between the size of the broad line region (BLR) and the bolometric luminosity, $R_{\mathrm{BLR}} \propto L^{1 / 2}$ (Kaspi et al. 1996; see also Kaspi et al. 2000 for a slightly steeper relation), and derived the SMBH mass for a number of quasars using the measured $\mathrm{H} \beta$ velocity dispersion and the continuum luminosity. We have compared the estimated SMBH masses for 9 Seyfert galaxies using Laor's method (but adopted the BLR velocity $V=(\sqrt{3} / 2) F W H M(\mathrm{H} \beta)$, as did Kaspi et al. 2000) with those obtained by reverberation mapping, and found that they agree well. This shows that using $\mathrm{SMBH}$ masses derived with different methods may not cause serious problems in our present work.

\section{Sample of Seyfert galaxies}

We selected 37 Seyfert galaxies from two well-studied nearby Seyfert samples, the Palomar optical spectroscopic survey of bright $\left(B_{\mathrm{T}} \leq 12.5 \mathrm{mag}\right)$, northern $\left(\delta>0^{\circ}\right)$ galaxies (Ho et al. 1995), including 21 Seyfert 1s and 28 Seyfert 2s (Ho et al. 1997a), the most complete and least biased available (Ho et al. 1997b; Ho \& Ulvestad 2001), and the CfA redshift Survey (Huchra et al. 1983) of galaxies with Zwicky magnitude $\leq 14.5$, including 33 Seyfert 1s and 15 Seyfert 2s (Huchra \& Burg 1992; Osterbrock \& Martel 1993). The Seyfert samples from these two surveys seem to complement one another, though the combined sample may not be complete (Ho \& Peng 2001).

Our Seyfert sample include 22 Seyfert 1 s and 15 Seyfert 2s in Palomar and CfA samples (see Table 1). These Seyfert galaxies have either the measured SMBH masses or the measured central velocity dispersions. Among them, three Seyfert 1s and two Seyfert 2s have dynamical SMBH masses (Ho 1999; Gebhardt et al. 2000a), and ten Seyfert 1s have SMBH masses measured by the reverberation mapping method (Wandel et al. 1999; Ho 1999). Another Nine Seyfert 1s and 13 Seyfert 2s have

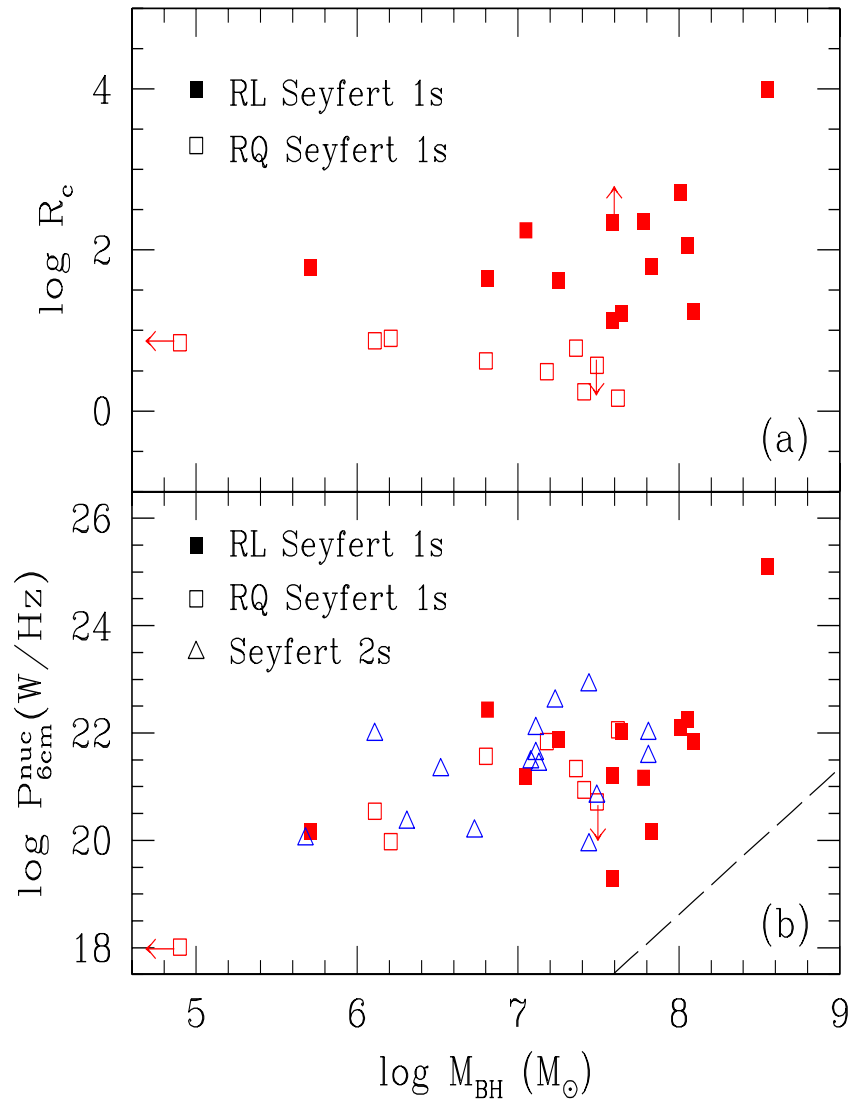

Fig. 2. The nuclear radio-loudness and the nuclear radio power against the black hole masses for Seyfert galaxies. The dashed line in the lower panel represents the tight correlation between the core radio power and the SMBH masses for nearby galaxies found by Franceschini et al. (1998).

measured central velocity dispersions but unknown SMBH masses (Nelson \& Whittle 1995). The SMBH masses can be estimated by Eq. (1) using the measured central velocity dispersions. Therefore, our sample consists of 37 Seyfert galaxies with derived $\mathrm{SMBH}$ masses (see Table 1).

\section{Radio properties and black hole masses of Seyfert galaxies}

The radio properties of Seyfert galaxies have been investigated in detail using the Very Large Array (VLA) at $3.6 \mathrm{~cm}$ by Kukula et al. (1995) for the CfA sample, and at $6 \mathrm{~cm}$ and $20 \mathrm{~cm}$ recently by Ho \& Ulvestad (2001) for the Palomar sample. In this paper, we adopt the radio data for Seyfert 1s from Ho \& Peng (2001) and the data for Seyfert 2s from Ho \& Ulvestad (2001) and Kukula et al. (1995). The $6 \mathrm{~cm}$ data for some sources in the CfA sample (Kukula et al. 1995) were extrapolated from the $3.6 \mathrm{~cm}$ data assuming $f_{\nu} \propto \nu^{-0.5}$.

Seyfert galaxies have been considered usually as radioquiet AGNs because most of them have lower radioloudness, defined as the ratio of the radio to optical luminosities, $R=L_{6 \mathrm{~cm}} / L_{B}$. However, a recently study of Ho \& Peng (2001) showed that though the nuclear 
Table 1. Black hole mass of a sample of Seyfert 1 and Seyfert 2 galaxies.

\begin{tabular}{|c|c|c|c|c|c|c|c|c|c|}
\hline Name & Type & $M_{B}^{\text {tot }}$ & $B-V$ & $M_{B}^{\text {nuc }}$ & $\begin{array}{r}\log P_{6 \mathrm{~cm}}^{\text {nuc }} \\
(\mathrm{W} / \mathrm{Hz})\end{array}$ & $\log R_{\mathrm{c}}$ & $M_{B}^{\mathrm{bul}}$ & $\begin{array}{c}M_{\mathrm{BH}} \\
\left(10^{7} M_{\odot}\right)\end{array}$ & Note* \\
\hline Mrk 279 & Sy1 & -20.92 & 0.69 & -20.55 & 22.06 & 0.16 & -20.31 & $4.2 \pm 1.7$ & $\mathrm{C}, 1, \mathrm{a}$ \\
\hline Mrk 335 & Sy1 & -21.48 & 0.34 & -18.18 & 21.57 & 0.62 & -20.62 & $0.63_{-0.17}^{+0.23}$ & $\mathrm{C}, 1$ \\
\hline Mrk 590 & Sy1 & -21.42 & 0.67 & -16.46 & 21.88 & 1.62 & -20.40 & $1.78_{-0.33}^{+0.44}$ & $\mathrm{C}, 1$ \\
\hline Mrk 817 & Sy1 & -21.03 & 0.40 & -17.81 & 22.02 & 1.21 & -18.49 & $4.4_{-1.1}^{+1.3}$ & $\mathrm{C}, 1$ \\
\hline NGC 3227 & Sy1 & -20.57 & 0.82 & -16.01 & 21.20 & 1.12 & -19.55 & $3.9_{-3.9}^{+2.1}$ & $\mathrm{P}, \mathrm{C}, 1$ \\
\hline NGC 3516 & Sy1 & -20.63 & 0.72 & -17.21 & 21.34 & 0.78 & -20.02 & $2.3 \pm 0.9$ & $\mathrm{P}, \mathrm{C}, 1, \mathrm{a}$ \\
\hline NGC 4051 & Sy1 & -20.38 & 0.67 & -14.97 & 20.54 & 0.87 & -18.41 & $0.13_{-0.08}^{+0.13}$ & $\mathrm{P}, \mathrm{C}, 1$ \\
\hline NGC 4151 & Sy1 & -20.16 & 0.71 & -19.18 & 21.84 & 0.49 & -18.93 & $1.53_{-0.89}^{+1.06}$ & $\mathrm{P}, \mathrm{C}, 1$ \\
\hline NGC 5548 & Sy1 & -20.97 & 0.62 & -17.29 & 21.84 & 1.24 & -20.11 & $12.3_{-1.8}^{+2.3}$ & $\mathrm{P}, \mathrm{C}, 1$ \\
\hline NGC 7469 & Sy1 & -21.32 & 0.38 & -17.78 & 22.43 & 1.64 & -20.30 & $0.65_{-0.65}^{+0.64}$ & $\mathrm{C}, 1$ \\
\hline NGC 3031 & Sy1 & -20.24 & 1.12 & -11.73 & 20.16 & 1.79 & -19.01 & $0.68_{-0.13}^{+0.07}$ & $\mathrm{P}, 2$ \\
\hline NGC 4258 & Sy1 & -20.13 & 0.77 & $>-8.17$ & 19.29 & $>2.34$ & -18.16 & $0.39 \pm 0.034$ & $\mathrm{P}, 2$ \\
\hline NGC 4395 & Sy1 & -16.51 & 0.53 & -8.69 & 18.01 & 0.85 & $>-16.51$ & $<0.008$ & $\mathrm{P}, \mathrm{C}, 2$ \\
\hline Mrk 530 & Sy1 & -21.48 & 0.72 & -16.27 & 22.24 & 2.06 & -19.94 & $11.26 \pm 8.49$ & $\mathrm{C}, 3$ \\
\hline Mrk 744 & Sy1 & -19.96 & 0.88 & -17.56 & 20.94 & 0.24 & -18.94 & $2.58 \pm 1.11$ & C, 3 \\
\hline NGC 1275 & Sy1 & -22.29 & 0.62 & -18.53 & 25.09 & 4.00 & $>-22.29$ & $35.88 \pm 11.61$ & $\mathrm{P}, 3$ \\
\hline NGC 3982 & Sy1 & -19.43 & 0.80 & -11.76 & 20.16 & 1.78 & -17.89 & $0.052 \pm 0.047$ & $\mathrm{P}, 3, \mathrm{~b}$ \\
\hline NGC 4388 & Sy1 & -19.51 & 0.80 & -13.17 & 21.19 & 2.24 & -17.97 & $1.12 \pm 0.80$ & $\mathrm{P}, 3, \mathrm{~d}$ \\
\hline NGC 4579 & Sy1 & -20.82 & 0.97 & -12.81 & 21.16 & 2.35 & -19.28 & $6.04 \pm 3.02$ & $\mathrm{P}, 3$ \\
\hline NGC 5252 & Sy1 & -20.93 & 1.00 & -14.23 & 22.10 & 2.72 & -20.32 & $10.20 \pm 6.84$ & $\mathrm{C}, 3$ \\
\hline NGC 5273 & Sy1 & -19.24 & 0.89 & -13.51 & 19.98 & 0.90 & -18.63 & $0.16 \pm 0.08$ & $\mathrm{P}, \mathrm{C}, 3$ \\
\hline NGC 6104 & Sy1 & -21.12 & 0.80 & -16.17 & $<20.72$ & $<0.57$ & -20.10 & $3.14_{-3.14}^{+3.50}$ & $\mathrm{C}, 3, \mathrm{~b}$ \\
\hline NGC 3079 & Sy2 & -21.14 & 0.87 & $\ldots$ & 21.97 & $\ldots$ & -16.91 & $1.3 \pm 0.4$ & $\mathrm{P}, 2$ \\
\hline NGC 1068 & Sy2 & -21.32 & 0.87 & $\ldots$ & 22.59 & $\ldots$ & -19.78 & $1.7_{-0.7}^{+1.3}$ & $\mathrm{P}, \mathrm{C}, 2$ \\
\hline NGC 1358 & Sy2 & -20.95 & 1.05 & $\ldots$ & 21.56 & $\ldots$ & -20.09 & $6.56 \pm 2.50$ & $\mathrm{P}, 3$ \\
\hline NGC 1667 & Sy2 & -21.52 & 0.80 & $\ldots$ & 21.99 & $\ldots$ & -18.98 & $6.56 \pm 4.65$ & $\mathrm{P}, 3, \mathrm{~d}$ \\
\hline NGC 2273 & Sy2 & -20.25 & 0.78 & $\ldots$ & 21.41 & $\ldots$ & -19.32 & $1.36 \pm 0.52$ & $\mathrm{P}, 3$ \\
\hline NGC 3185 & Sy2 & -18.99 & 0.80 & $\ldots$ & 20.02 & $\ldots$ & -17.97 & $0.048_{-0.048}^{+0.074}$ & $\mathrm{P}, 3$ \\
\hline NGC 5194 & Sy2 & -20.76 & 0.91 & $\ldots$ & 20.17 & $\ldots$ & -18.79 & $0.54_{-0.54}^{+0.640}$ & $\mathrm{P}, 3$ \\
\hline NGC 7743 & Sy2 & -19.78 & 0.91 & $\ldots$ & 20.33 & $\ldots$ & -19.05 & $\begin{array}{l}0.20_{-0.20}^{+0.23} \\
\end{array}$ & $\mathrm{P}, 3$ \\
\hline Mrk 573 & Sy2 & -20.32 & 0.83 & $\ldots$ & 21.61 & $\ldots$ & -19.71 & $1.31 \pm 0.80$ & C, 3 \\
\hline NGC 3362 & Sy2 & -21.99 & 0.80 & $\ldots$ & 21.31 & $\ldots$ & -19.45 & $0.33_{-0.32}^{+0.48}$ & $\mathrm{C}, 3, \mathrm{~b}$ \\
\hline NGC 5929 & Sy2 & -20.48 & 0.80 & $\ldots$ & 21.46 & $\ldots$ & -19.24 & $1.21 \pm 0.62$ & $\mathrm{C}, 2, \mathrm{~b}$ \\
\hline NGC 7682 & Sy2 & -20.23 & 0.80 & $\ldots$ & 22.08 & $\ldots$ & -19.00 & $1.31 \pm 0.85$ & $\mathrm{C}, 3, \mathrm{~b}$ \\
\hline NGC 5283 & Sy2 & -19.40 & 0.92 & $\ldots$ & 20.82 & $\ldots$ & -18.79 & $3.14 \pm 1.40$ & $\mathrm{C}, 3$ \\
\hline NGC 5695 & Sy2 & -20.50 & 0.95 & $\ldots$ & 19.91 & $\ldots$ & -19.48 & $2.76 \pm 0.99$ & $\mathrm{C}, 3$ \\
\hline NGC 7674 & Sy2 & -21.77 & 0.83 & $\ldots$ & 22.89 & $\ldots$ & -19.81 & $2.76_{-2.76}^{+2.89}$ & $\mathrm{C}, 3$ \\
\hline
\end{tabular}

* Notes: (C), object in CfA Seyfert sample; (P), object in Palomar Seyfert sample; (1), $M_{\mathrm{BH}}$ measured by reverberation mapping; (2), $M_{\mathrm{BH}}$ measured by gas/stellar dynamics; (3), $M_{\mathrm{BH}}$ obtained in this paper using the $M_{\mathrm{BH}}-\sigma$ relation; (a), uncertainty of $M_{\mathrm{BH}}$ not available in literature and assumed to be $40 \%$; (b), $B-V$ value not available in literature and assumed to be $0.80 ;(d)$, uncertainty of $\sigma$ not available in literature and assumed to be $15 \%$.

radio power for Seyfert 1 galaxies on average accounts for about $75 \%$ of the total radio emission, the nuclear optical luminosity, measured by high-resolution optical images, accounts for merely $0.01 \%$ of the integrated light. If the radio-loudness is measured by the nuclear radio and optical luminosities, most Seyfert 1s are in the category of radio-loud AGNs (with radio-loudness larger than 10). In Table 1, we listed the total and nuclear absolute $B$ magnitudes $\left(M_{B}^{\text {tot }}\right.$ and $\left.M_{B}^{\text {nuc }}\right)$ and nuclear radio power $\left(P_{6} \mathrm{~cm}\right)$ for Seyfert galaxies. The nuclear radio-loudness, $R_{\mathrm{c}}$, is calculated by $R_{\mathrm{c}}=L_{6 \mathrm{~cm}}^{\text {nuc }} / L_{B}^{\text {nuc }}$. The Hubble constant $H_{0}=75 \mathrm{~km} \mathrm{~s}^{-1} \mathrm{Mpc}^{-1}$ and the deceleration parameter of $q_{0}=0.5$ were adopted. For Seyfert $2 \mathrm{~s}$, the measurements of their nuclear optical magnitudes are not available in the literature.
Figure 2 shows the relation of SMBH masses with the nuclear radio-loudness of Seyfert 1s and the radio power of Seyfert galaxies listed in Table 1. It is clear that the radioloud Seyfert 1s with larger nuclear radio-loudness seem to host more massive black holes. The similar tendency has been found for PG quasars by Laor (2000). From Fig. 2b we see a trend that Seyfert galaxies having a larger radio power perhaps host a more massive black hole than those of less radio power. With the same black hole mass, Seyfert galaxies seem to have 100 to 1000 times greater radio power than normal galaxies. Laor (2000) has shown that PG quasars also depart from such a correlation for nearby galaxies, with the radio luminosity of quasars being $10^{4}$ larger at a given $M_{\mathrm{BH}}$. The difference of radio 


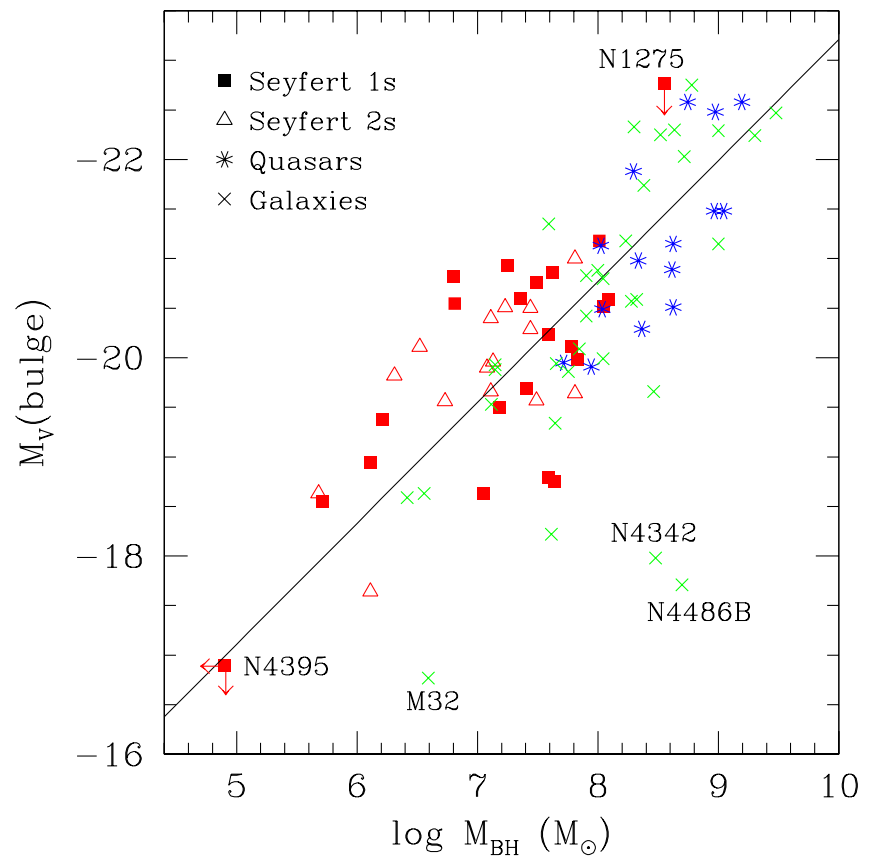

Fig. 3. Correlation of $V$-band bulge absolute magnitudes and SMBH masses for Seyfert galaxies, quasars and normal galaxies. The solid line represents the least $\chi^{2}$ fit to all objects by considering typical measurement errors of both parameters.

luminosities of quasars, Seyfert galaxies and nearby galaxies may be simply due to different levels of nuclear activity.

\section{Black hole masses and bulge luminosities}

The correlation of black hole mass and galactic bulge luminosity found for nearby galaxies implies a relationship between the black hole and bulge masses (Kormendy \& Richstone 1995; Magorrian et al. 1998; Ho 1999). However, it is not clear whether the ratio between black hole and bulge masses remains constant for all kinds of objects. Laor (2001) recently checked the correlation between the estimated black masses and bulge luminosities for 15 nearby quasars and 9 Seyfert galaxies, and found that they probably follow the same $M_{\mathrm{BH}}$-bulge luminosity relation as for 16 nearby galaxies. This suggests a universal nonlinear relation between the estimated black masses and bulge luminosities, $M_{\mathrm{BH}} \propto M_{\text {bulge }}^{1.54 \pm 0.15}$, for both normal and active galaxies. Therefore, $M_{\mathrm{BH}} / M_{\text {bulge }}$ ratio is not constant for galaxies with different bulge luminosities. However, this needs to be confirmed by larger samples including both normal and active galaxies.

\subsection{Our sample of Seyfert galaxies}

We use the 37 Seyfert galaxies with derived SMBH masses in our sample to check the $M_{\mathrm{BH}} / M_{\text {bulge }}$ ratio. The absolute total $B$ magnitudes $\left(M_{B}^{\text {tot }}\right)$ for our Seyfert sample (see Table 1) were taken from Ho et al. (1997b) and Whittle (1992) (adapted to $H_{0}=75 \mathrm{~km} \mathrm{~s}^{-1} \mathrm{Mpc}^{-1}$ ). The absolute bulge $B$ magnitudes $\left(M_{B}^{\text {bulge }}\right)$ were obtained based on the relation between $M_{B}^{\text {bulge }}$ and $M_{B}^{\text {tot }}$ (Simien
$\&$ de Vaucouleurs 1986) and the Hubble stages (defined in de Vaucouleurs et al. 1976) of the host galaxies. The $B-V$ colors were taken from Véron-Cetty \& Véron (2000). In order to compare our result with those obtained for quasars and nearby galaxies, we convert $M_{B}^{\text {bulge }}$ of Seyfert galaxies to the absolute bulge $V$ magnitude, $M_{V}^{\text {bulge }}$ (adapted to $H_{0}=80 \mathrm{kms}^{-1} \mathrm{Mpc}^{-1}$ ) by assuming that $B-V$ color in the bulge is nearly the same as the total $B-V$ color of Seyfert galaxy. In Fig. 3 we show the relation between the SMBH masses and bulge $V$-band absolute magnitudes for Seyfert galaxies and the comparison of this relation with those for quasars and nearby galaxies. The SMBH masses of 15 quasars and the absolute $V$ magnitudes of their inner host were taken from Laor $(1998,2001)^{1}$. The SMBH masses determined by stellar and gas dynamics for 33 nearby galaxies were taken from Kormendy \& Gebhardt (2001) (we omitted M 81, a Seyfert 1 already in our Seyfert sample, and 3 objects with black hole masses measured by maser dynamics). Their absolute bulge $V$ magnitudes were derived from $B$-band absolute bulge magnitudes by adopting a standard bulge color, $B-V=0.94$ (Worthey 1994). It is evident that both Seyfert and nearby galaxies, as well as quasars, seem to follow the same $M_{V}^{\text {bulge }}-M_{\mathrm{BH}}$ relation. Note that unlike others, nearby galaxies NGC 4342, NGC 4486B and M 32 deviate significantly from the $M_{V}^{\text {bulge }}-M_{\mathrm{BH}}$ relation. These offset galaxies are usually fainter because the outer regions of them may have been stripped away in the tidal interactions with more massive companions (Faber 1973). These three galaxies, together with two Seyfert 1 galaxies, NGC 1275 with peculiar Hubble type and NGC 4395 with only the upper limit of measured SMBH mass, are not included in our statistical studies. The measurement errors of $M_{\mathrm{BH}}$ of our Seyfert sample were listed in Table 1, and those of normal galaxies were adopted from Kormendy \& Gebhardt (2001). For nearby quasars, such errors are not available in Laor (1998) and assumed to be $60 \%$. The measurement errors of $M_{V}^{\text {bulge }}$ were seldom mentioned in the literature and thus are difficult to estimate. These errors for Seyfert galaxies were allocated to be 0.5, 0.75 and 1.0 mag respectively based on their quality assessment factors (Whittle 1992). Those of normal galaxies and nearby quasars were adopted to be 0.5 and $0.75 \mathrm{mag}$, respectively. Taking into account these "typical" errors of $M_{V}^{\text {bulge }}$ and $M_{\mathrm{BH}}$, the least $\chi^{2}$ fit for 35 Seyfert galaxies, 15 quasars and 30 normal galaxies gives:

$$
M_{V}^{\text {bulge }}=-11.01 \pm 0.78-(1.22 \pm 0.10) \log \left(M_{\mathrm{BH}} / M_{\odot}\right)
$$

The Spearman rank-order correlation coefficient is $r_{\mathrm{S}}=$ -0.76 , which has a probability of $P_{\mathrm{r}}=3.3 \times 10^{-16}$ occuring by chance. Considering the errors in both parameters, we adopted a bootstrap method to estimate the uncertainty of this correlation, and obtained the mean

\footnotetext{
1 Because we used BLR velocity dispersion as $V=$ $(\sqrt{3} / 2) F W H M(\mathrm{H} \beta)$, the SMBH masses of 15 quasars are smaller by a factor of 0.75 than those given by Laor (1998).
} 
correlation coefficient $\left\langle r_{\mathrm{S}}\right\rangle=-0.63 \pm 0.05$. Using the standard relation

$M_{V}^{\text {bulge }}=4.83-2.5 \log \left(L_{\text {bulge }} / L_{\odot}\right)$,

and the relation between the bulge mass and luminosity (Magorrian et al. 1998),

$\log \left(M_{\text {bulge }} / M_{\odot}\right)=-1.11+1.18 \log \left(L_{\text {bulge }} / L_{\odot}\right)$,

we then can get from Eq. (2),

$\log \left(M_{\mathrm{BH}} / M_{\text {bulge }}\right)=-11.06 \pm 1.11$

$$
+(0.74 \pm 0.14) \log \left(M_{\text {bulge }} / M_{\odot}\right) \text {. }
$$

This gives $M_{\mathrm{BH}} \propto M_{\text {bulge }}^{1.74 \pm 0.14}$. The fitting for a sample of 35 Seyfert galaxies and 30 normal galaxies alone gives almost identical results. Laor (2001) recently found $M_{\mathrm{BH}} \propto M_{\text {bulge }}^{1.54 \pm 0.15}$ for a sample of objects including 9 Seyfert galaxies and 15 quasars. Our result shows that this nonlinear relation is more evident from a larger sample including more Seyfert galaxies. Equation (5) also shows that $M_{\mathrm{BH}} / M_{\text {bulge }}$ is about $0.02 \%$ when $M_{\mathrm{BH}}=$ $10^{6} M_{\odot}$, and is $0.3 \%$ when $M_{\mathrm{BH}}=10^{9} M_{\odot}$. This clearly indicates that galaxies with more massive black holes have a larger $M_{\mathrm{BH}} / M_{\text {bulge }}$ ratio.

\subsection{Seyfert sample in reverberation mapping studies}

We noted that the above universal $M_{V}^{\text {bulge }}-M_{\mathrm{BH}}$ relation (Eq. (2)) is contrary to the result obtained by Wandel (1999), who found that Seyfert 1 galaxies significantly depart from the $L_{\text {bulge }}-M_{\mathrm{BH}}$ relations of quasars and nearby galaxies (Laor 1998; Magorrian et al. 1998). However, Mclure \& Dunlop (2001) used the decomposed bulge luminosities of the same sample of Seyfert galaxies as in Wandel (1999) and found no evidence for a different $L_{\text {bulge }}-M_{\mathrm{BH}}$ relation from quasars. We noted that Wandel (1999) took the absolute bulge $B$ magnitude, $M_{B}^{\text {bulge }}$, for Seyfert galaxies from Whittle (1992) (adopting $H_{0}=50 \mathrm{~km} \mathrm{~s}^{-1} \mathrm{Mpc}^{-1}$ ) and calculated the bulge luminosity from $M_{B}^{\text {bulge }}$ with the standard expression for $M_{V}^{\text {bulge }}$ (see Eq. (3)) by assuming $M_{B}^{\text {bulge }} \simeq M_{V}^{\text {bulge }}$ for Seyfert $1 \mathrm{~s}$. We carefully checked these points and re-derived the absolute bulge $V$ magnitude of 17 Seyfert 1 galaxies with SMBH masses measured by reverberation mapping (Wandel et al. 1999), using $H_{0}=80 \mathrm{~km} \mathrm{~s}^{-1} \mathrm{Mpc}^{-1}$ and assuming the bulge $B-V$ color being nearly the same as the $B-V$ color of the whole galaxy. The $M_{V}^{\text {bulge }}-M_{\mathrm{BH}}$ relation of 17 Seyfert 1 galaxies, as compared with those for 15 quasars (Laor 1998, 2001) and 30 nearby galaxies with $M_{\mathrm{BH}}$ measured by stellar and gas dynamics (Kormendy \& Gebhardt 2001), is shown in Fig. 4. Allocating the "typical" uncertainties of $M_{V}^{\text {bulge }}$ and $M_{\mathrm{BH}}$ as we did above, the least $\chi^{2}$ fit for 62 objects gives,

$M_{V}^{\text {bulge }}=-10.00 \pm 0.96-(1.34 \pm 0.12) \log \left(M_{\mathrm{BH}} / M_{\odot}\right) .(6)$

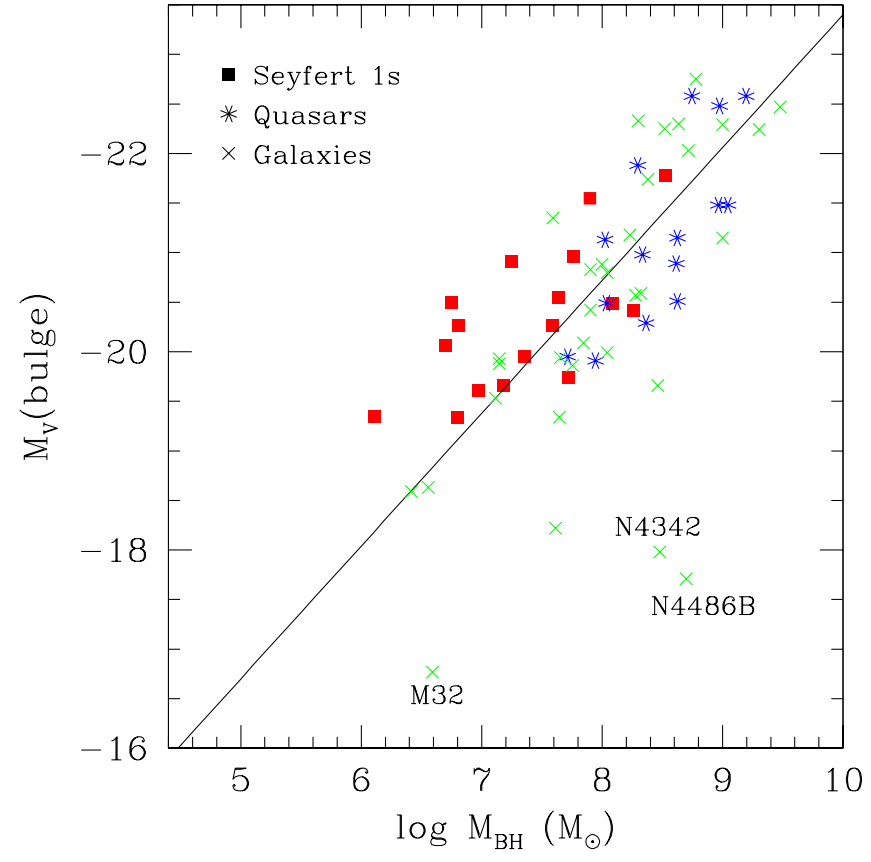

Fig. 4. Correlation between the $V$-band absolute bulge magnitudes and black hole masses for quasars, normal galaxies and Seyfert 1 galaxies with black hole masses only measured by reverberation mapping. The solid line represents the least $\chi^{2}$ fit to all objects by considering typical measurement errors of both parameters.

Considering the errors in both parameters, we estimated the mean correlation coefficient as $\left\langle r_{\mathrm{S}}\right\rangle=-0.63 \pm 0.06$. This result is consistent with that found above (see Eq. (2)) and also indicates a nonlinear $M_{\mathrm{BH}}-M_{\text {bulge }}$ relation.

\subsection{Nelson and Whittle's sample of Seyfert galaxies}

In this section we explore the Seyfert sample in Nelson \& Whittle (1995), where the measurements of nuclear velocity dispersions of about 70 Seyfert galaxies were reported. After excluding several LINERs and normal galaxies, we got 33 Seyfert 1 s and 32 Seyfert 2s. We estimated the SMBH masses of these Seyfert galaxies using the $M_{\mathrm{BH}^{-}} \sigma$ relation (Eq. (1)). The total radio power at $5 \mathrm{GHz}$ of them was calculated from the $1.4 \mathrm{GHz}$ data in Nelson \& Whittle (1995) by assuming $f_{\nu} \propto \nu^{-0.5}$ and $H_{0}=80 \mathrm{~km} \mathrm{~s}^{-1} \mathrm{Mpc}^{-1}$. The radio-loudness was calculated by the $5 \mathrm{GHz}$ radio luminosity and the $B$-band optical luminosity. The $V$-band bulge absolute magnitude was estimated from the $B$-band bulge absolute magnitude (adapted to $H_{0}=80 \mathrm{~km} \mathrm{~s}^{-1} \mathrm{Mpc}^{-1}$ ) in Nelson \& Whittle (1995) by assuming the $B-V$ color of the bulge being the same as the total $B-V$ color (taken from Véron-Cetty \& Véron 2000) of Seyfert galaxy.

The radio-loudness and total radio power against the SMBH masses for 29 Seyfert 1 s and 25 Seyfert $2 \mathrm{~s}$ with available radio data in the sample of Nelson \& Whittle (1995) are plotted in Fig. 5. Now we see that most Seyfert galaxies seem to be radio quiet $(R<10)$ when we adopted 


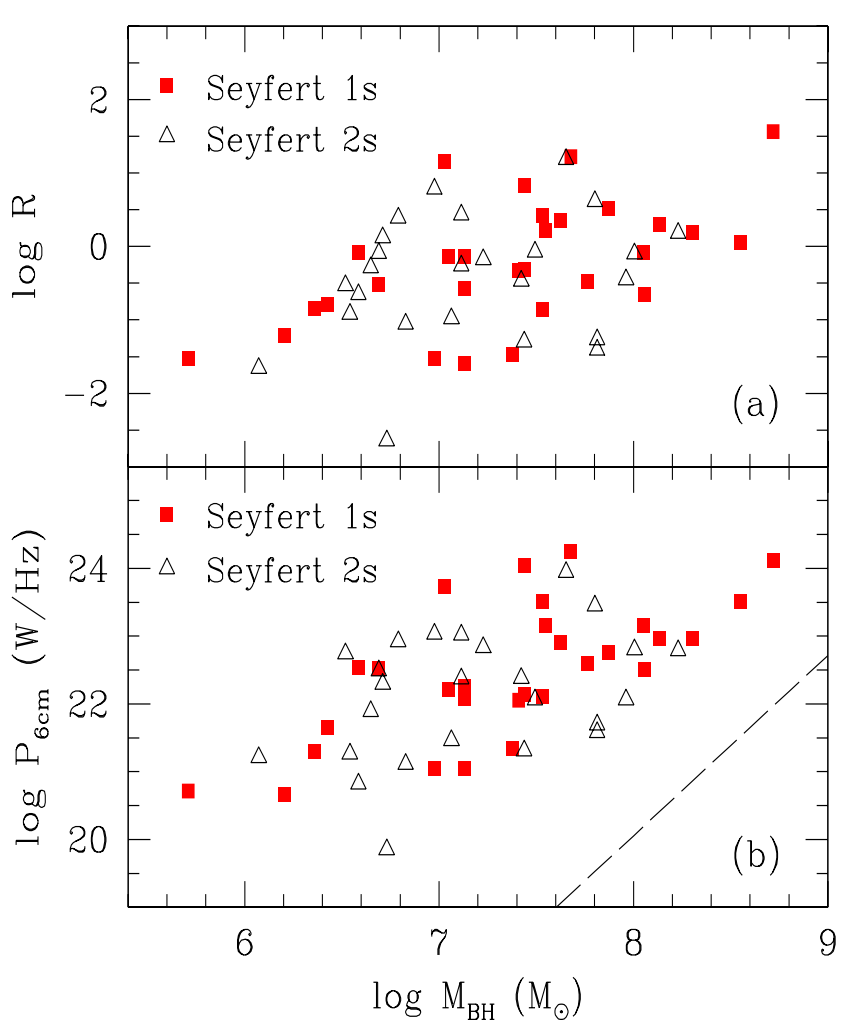

Fig. 5. The radio-loudness a) and radio power $\mathbf{b}$ ) against the black hole masses for Seyfert galaxies in the sample of Nelson \& Whittle (1995). The dashed line in b) represents the tight correlation between total radio power and SMBH masses found by Franceschini et al. (1998) for nearby galaxies.

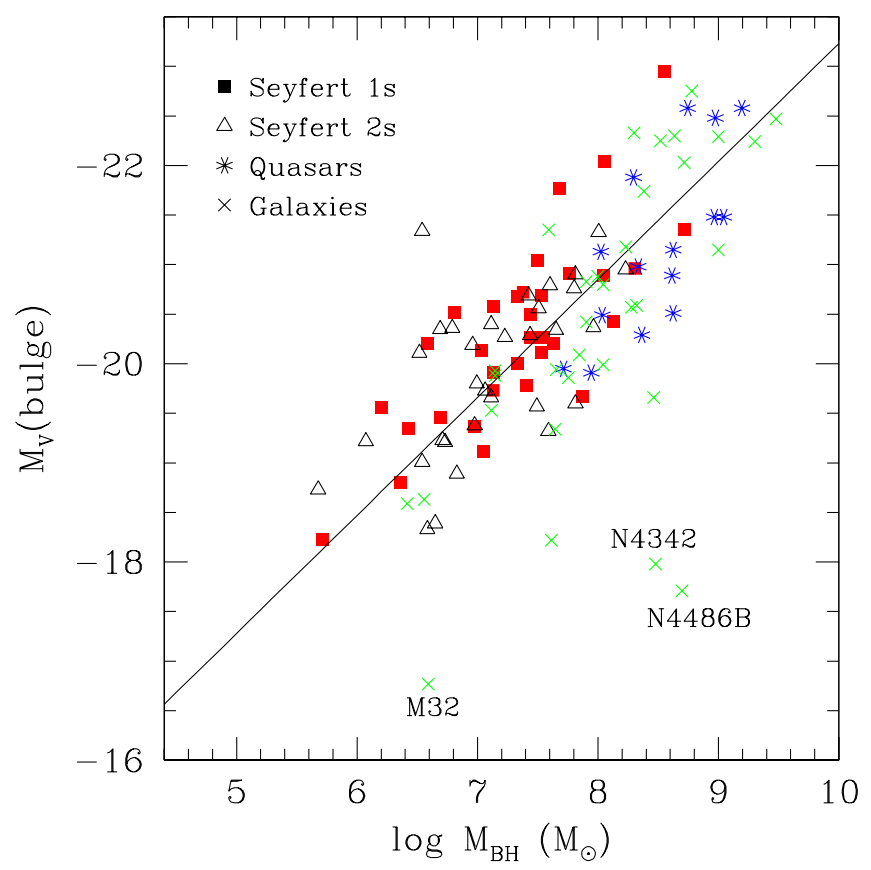

Fig. 6. Correlation of $V$-band absolute bulge magnitudes and black hole masses for quasars, normal galaxies and Seyfert galaxies in the sample of Nelson \& Whittle (1995). The solid line represents the least $\chi^{2}$ fit to all objects. The typical measurement errors of both parameters were considered. the total radio and optical luminosities to calculate the radio-loudness. There is a weak tendency that Seyfert galaxies with larger radio-loudness have larger SMBH masses. A comparison with the result in Fig. 2a indicates that the nuclear radio-loudness may be more fundamental and reflect the nature of central engine of Seyfert galaxies. From Fig. 5b we see that there is a strong correlation between the total radio power and the SMBH mass, though the scatters are large. This confirms again the previous result that AGNs with larger radio power may host more massive SMBHs (Franceschini et al. 1998; McLure et al. 1999). However, as is shown in Fig. 2, Seyfert galaxies depart significantly from the tight relation between the radio power and SMBH mass found for normal galaxies by Franceschini et al. (1998).

The relation between $V$-band bulge luminosities and SMBH masses for 33 Seyfert 1 s and 32 Seyfert $2 \mathrm{~s}$ in the sample of Nelson \& Whittle (1995) is shown in Fig. 6. It again indicates a significant universal relation between $V$-band bulge luminosities and SMBH masses for both Seyfert galaxies and quasars, as well as nearby galaxies. Adopting the "typical" uncertainties of $M_{V}^{\text {bulge }}$ and $M_{\mathrm{BH}}$ as we did above, the least $\chi^{2}$ fit for 65 Seyfert galaxies, 15 quasars and 30 nearby galaxies gives,

$M_{V}^{\text {bulge }}=-11.33 \pm 0.66-(1.19 \pm 0.09) \log \left(M_{\mathrm{BH}} / M_{\odot}\right)$. (7)

The simple Spearman rank-order correlation coefficient is $r_{\mathrm{S}}=-0.76$, which has a probability of $P_{\mathrm{r}}=1.1 \times 10^{-21}$ occurring by chance. Considering the errors in both parameters, we estimated the mean correlation coefficient as $\left\langle r_{\mathrm{S}}\right\rangle=-0.61 \pm 0.04$. This result is also almost identical to what we found for the sample including 35 Seyfert galaxies. Therefore, the nonlinear $M_{\mathrm{BH}}-M_{\text {bulge }}$ relation is confirmed by the substantially enlarged Seyfert sample.

\subsection{Effects of larger systematic errors}

A significant correlation between black hole mass and bulge luminosity was obtained above by considering the "typical" errors of both parameters. These errors are mainly due to the measurement uncertainties of bulge magnitude and black hole mass. However, the systematic errors are probably substantially larger for both bulge magnitude and black hole mass. For example, if $M_{\mathrm{BH}}$ is determined by reverberation mapping, the systematic errors caused by the unknown BLR geometry, inclination may be as large as a factor of 3 or more (Krolik 2001). Using the $M_{\mathrm{BH}}-\sigma$ relation to estimate $M_{\mathrm{BH}}$ also has larger systematic errors due to the uncertainty of the slope of this relation. In addition, in deriving bulge magnitude from galaxy magnitude for Seyfert galaxies we adopted a statistical relation given by Simien \& de Vaucouleurs (1986). The systematic errors caused by applying this relation are probably quite large and are difficult to estimate quantitatively.

In order to estimate the effects of possible larger systematic errors of black hole mass and bulge magnitude on our result, we adopted a bootstrap method by adding 
the systematic errors into the uncertainties of both parameters. Assuming the possible systematic errors for bulge absolute magnitude being $1 \mathrm{mag}$ and those for black hole mass being $90 \%$ for all 35 Seyfert galaxies, 15 quasars and 30 normal galaxies, we obtained the average correlation coefficient using the bootstrap approach as being $\left\langle r_{\mathrm{S}}\right\rangle=-0.40 \pm 0.09$, which indicates a moderately significant correlation. The minimum chi-square fit considering both measurement and systematic errors in both parameter gives, $M_{V}^{\text {bulge }}=-10.49 \pm 1.77-(1.29 \pm$ $0.22) \log \left(M_{\mathrm{BH}} / M_{\odot}\right)$. Comparing with the result we obtained in Eq. (2), we found the slope of this relation does not change very much, though the uncertainty of the slope is doubled when the larger systematic errors are considered. The correlation coefficient also substantially decreases and its uncertainty increases accordingly. However, these changes are quite limited and have no significant effects on the result we have obtained.

\section{Discussions}

Our results obtained for a larger sample of Seyfert galaxies show that AGNs with a larger nuclear radio-loudness seem to have more massive black holes. This conclusion was obtained recently by Laor (2000) for nearby quasars and was supported by our study for Seyfert galaxies. Our results also strengthen the argument made by Ho \& Peng (2001) that the majority of Seyfert 1 s are essentially radio-loud AGNs. The total radio power of Seyfert galaxies increases with the black hole mass. At a given $M_{\mathrm{BH}}$, quasars and Seyfert galaxies seem to have greater radio power than that of nearby galaxies. These differences may simply be due to the different level of nuclear activity for different kinds of galaxies. A recent study using the quasars from the FIRST Bright Quasar Survey found evidence for the dependence of radio-luminosity on accretion rate and SMBH mass (Lacy et al. 2001). This may help us to understand the origin of scatters in the relation between the radio power and SMBH mass. If we describe the nuclear activity of galaxies using the accretion rate $\dot{M}$, the difference of radio power at a given $M_{\mathrm{BH}}$ may show that quasars and Seyfert galaxies have larger $\dot{M}$ than nearby galaxies. This seems also consistent with the picture that the accretion process in these systems may be different (Fabian \& Rees 1995; Di Matteo \& Fabian 1996). Most likely ADAFs with very low accretion rate exist in the nuclei of nearby galaxies, while quasars and most AGNs probably host standard geometrically thin accretion disks with higher accretion rate. However, the radio emissions from radioquiet AGNs and nearby galaxies are not well understood at present. Whether they are from ADAFs (Narayan et al. 1998) or from weak jets (Falcke \& Biermann 1996, 1999) still remains uncertain. For radio-loud AGNs, the radio emissions are thought to be mainly from the jet and are probably related to the magnetic fields or black hole spin (Blandford \& Payne 1982; Blandford \& Znajek 1977). If the radio emissions correlate with the black hole mass and accretion rate, we need to explain the possible relations of these parameters with magnetic fields and black hole spin. However, no satisfactory theory can provide clear physics about these relations at present.

Using the sample including 37 Seyfert galaxies in two well-defined bright Seyfert samples, we studied the correlations of black hole masses with $V$-band bulge absolute magnitudes and bulge masses for active and normal galaxies. We find that the correlation between the black hole masses and the $V$-band absolute bulge magnitudes for Seyfert galaxies is almost consistent with those found for quasars and nearby galaxies. The combined sample of 37 Seyfert galaxies, 15 quasars and 30 nearby galaxies seem to follow a universal nonlinear relation between the black hole and bulge masses, $M_{\mathrm{BH}} \propto M_{\text {bulge }}^{1.74 \pm 0.14}$, which is slightly steeper than that found recently by Laor (2001) for a sample including 9 Seyfert galaxies. Our results support the suggestion that the ratio of $M_{\mathrm{BH}}$ and $M_{\text {bulge }}$ is not constant and galaxies with larger bulge luminosities or more massive SMBH probably have a larger $M_{\mathrm{BH}} / M_{\text {bulge }}$ ratio. Including 65 Seyfert galaxies in the larger sample of Nelson \& Whittle (1995) led to almost the same result. In fact, the nonlinear relation between $M_{\mathrm{BH}}$ and $M_{\text {bulge }}$ has been predicted by some theories and supported by some recent studies. For example, using a collapse model for black hole and bulge formation, Adams et al. (2001) predicted $M_{\mathrm{BH}} / M_{\text {bulge }} \propto \sigma$, which gives $M_{\mathrm{BH}} / M_{\text {bulge }} \propto$ $M_{\mathrm{BH}}^{1 / 4}$ if $M_{\mathrm{BH}} \propto \sigma^{4}$. This states clearly that the ratio of black hole and bulge masses is not constant and can be larger for galaxies with more massive SMBHs. A similar result can be obtained by exploring the model of Wang et al. (2000) who derived $M_{\mathrm{BH}} / M_{\text {bulge }} \propto \sigma^{1.4}$. In addition, recent studies on narrow line Seyfert 1s showed that their mean $M_{\mathrm{BH}} / M_{\text {bulge }}$ ratio is significantly smaller than that for normal Seyfert galaxies (Mathur et al. 2001), which is also consistent with our results because narrowline Seyfert 1s probably have smaller $M_{\mathrm{BH}}$ than normal Seyfert galaxies (Boller et al. 1996).

Finally we would like to mention that the tight correlation between the black hole mass and bulge luminosity is not surprising and is actually expected from some existing well-known relations. From the $M_{\mathrm{BH}^{-}} \sigma$ relation and Faber-Jackson relation between the bulge luminosity and $\sigma$ (Faber \& Jackson 1976), we certainly expect a relation between black hole mass and bulge luminosity. The detailed studies of this relation and the $M_{\mathrm{BH}}-M_{\text {bulge }}$ relation are necessary because these relations are probably more fundamental than the Faber-Jackson relation and are more closely related to the physics of black hole and galaxy formation. We noticed that our result on the $M_{\mathrm{BH}}-M_{\text {bulge }}$ relation implies a rather flatter $L_{\mathrm{bulge}}-\sigma$ relation than the Faber-Jackson relation. This seems to be supported by the finding of Nelson \& Whittle (1996). However, the slope of the $L_{\mathrm{bulge}}-\sigma$ relation depends sensitively on the statistical method, sample selection and uncertainties of both parameters. In addition, in deriving the $M_{\mathrm{BH}} / M_{\text {bulge }}$ ratio, we adopted the same bulge mass-to-light ratio for Seyfert galaxies as for nearby 
galaxies obtained by Magorrian et al. (1998). Such a ratio may be smaller for Seyfert galaxies (Whittle 1992). Introducing a smaller bulge mass-to-light ratio may affect our results. We expect that further high quality observations on Seyfert galaxies, normal galaxies and quasars using the Hubble Space Telescope and larger ground-based telescopes could diminish the uncertainties in measuring the galactic bulge properties and central black hole masses of these objects. These efforts will undoubtedly help us to understand better the physics of formation and evolution of SMBHs and galaxies.

Acknowledgements. We are very grateful to the referee, Todd Boroson, for helpful comments and suggestions. We thank Peter Biermann, Xinwu Cao, Jiansheng Chen, Zugan Deng, Jun Ma, Tinggui Wang, Hong Wu, Xiaoyang Xia and Suijian Xue for stimulating discussions. This work was partially supported by the Pandeng Project, the National Natural Science Foundation, and the National Key Basic Research Science Foundation (NKBRSF G19990752) in China.

\section{References}

Adams, F. C., Graff, D. G., \& Richstone, D. O. 2001, ApJ, 551, 31

Begelman, M. C., Blandford, R. D., \& Rees, M. J. 1984, Rev. Mod. Phys., 56, 255

Blandford, R. D., \& Payne, D. G. 1982, MNRAS, 199, 883

Blandford, R. D., \& Znajek, R. L. 1977, MNRAS, 179, 433

Blundell, K. M., \& Beasley, A. J. 1998, MNRAS, 299, 165

Boller, Th., Brandt, W. N., \& Fink, H. 1986, A\&A, 305, 53

Boroson, T. A., \& Green, R. F. 1992, ApJS, 80, 109

de Vaucouleurs, G., de Vaucouleurs, A., \& Corwin, J. R. 1976, Second Reference Catalogue of Bright Galaxies (Austin: University of Texas Press)

Di Matteo, T., \& Fabian, A. C. 1996, MNRAS, 286, 393

Faber, S. M. 1973, ApJ, 179, 423

Faber, S. M., \& Jackson, R. E. 1976, ApJ, 204, 668

Fabian, A. C., \& Rees, M. J. 1995, MNRAS, 277, L55

Falcke, H., \& Biermann, P. L. 1996, A\&A, 308, 321

Falcke, H., \& Biermann, P. L. 1999, A\&A, 342, 49

Ferrarese, L., \& Merritt, D. 2000, ApJ, 539, L9

Ferrarese, L., Pogge, R. W., Peterson, B. M., et al. 2001, ApJ, $555, \mathrm{~L} 79$

Franceschini, A., Vercellone, S., \& Fabian, A. C. 1998, MNRAS, 297, 817

Gebhardt, K., Bender, R., Bower, G. et al. 2000a, ApJ, 539, L13

Gebhardt, K., Kormendy, J., Ho, L. C. et al. 2000b, ApJ, 543, L5

Genzel, R., Eckart, A., Ott, T., \& Eisenhauer, F. 1997, MNRAS, 291, 219

Ghez, A., Klein, B. L., Morris, M., \& Becklin, E. E. 1998, ApJ, 509, 678

Haehnelt, M. G., Natarajan, P., \& Rees, M. J. 1998, MNRAS, 300,817

Haehnelt, M. G., \& Rees, M. J. 1993, MNRAS, 263, 168

Haiman, Z., \& Loeb, A. 1998, ApJ, 503, 505

Ho, L. C. 1999, in Observational Evidence for Black Holes in the Universe, ed. S. K. Charkrabarti (Dordrecht: Kluwer), 157

Ho, L. C., Filippenko, A.V., \& Sargent, W. L. W. 1995, ApJS, 98,477
Ho, L. C., Filippenko, A.V., \& Sargent, W. L. W. 1997a, ApJS, 112,315

Ho, L. C., Filippenko, A. V., \& Sargent, W. L. W. 1997b, ApJ, 487,568

Ho, L. C., \& Peng, C. Y. 2001, ApJ, 555, 650

Ho, L. C., \& Ulvestad, J. S. 2001, ApJS, 133, 77

Huchra, J. P., \& Burg, R. 1992, ApJ, 393, 90

Huchra, J. P., Dacis Latham, D., \& Tonry, J. 1983, ApJS, 52, 89

Kaspi, S., Smith, P. S., Maoz, D., Netzer, H., \& Jannuzi, B. T. 1996, ApJ, 471, L75

Kaspi, S., Smith, P. S., Netzer, H., et al. 2000, ApJ, 533, 631

Kato, S., Fukue, J., \& Mineshige, S. 1998, Black Hole Accretion Disks (Kyoto University Press)

Kauffmann, G., \& Haehnelt, M. G. 2000, MNRAS, 311, 576

Kormendy, J., \& Richstone, D. 1995, ARA\&A, 33581

Kormendy, J., \& Gebhardt, K. 2001, Proceedings of The 20th Texas Symposium on Relativistic Astrophysics, ed. H. Martel \& J. C. Wheeler, in press [astro-ph/0105230]

Krolik, J. H. 2001, ApJ, 551, 72

Kukula, M. J., Pedlar, A., Baum, S. A., \& O'Dea, C. P. 1995, MNRAS, 276, 1262

Lacy, M., Laurent-Muehleisen, S. A., Ridgway, S. E., Becker R. H., \& White, R. L., ApJ, 551, L17

Laor, A. 1998, ApJ, 505, L83

Laor, A. 2000, ApJ, 543, L111

Laor, A. 2001, ApJ, 553, 677

Lyden-Bell, D. 1969, Nature, 223, 690

Magorrian, J., Tremaine, S., Richstone, D., et al. 1998, AJ, 115,2285

Mathur, S., Kuraszkiewicz, J., \& Czerny, B. 2001, New Astron., 6,321

McLure, R. J, \& Dunlop, J. S. 2001, MNRAS, 327, 199

McLure, R. J, Kukula, M. J., Dunlop, J. S., et al. 1999, MNRAS, 308, 377

Merritt, D., \& Ferrarese, L. 2001, ApJ, 547, 140

Narayan, R., Mahadevan, R., \& Quataert, E. 1998, in Theory of Black Hole Accretion Disks, ed. M. A. Abramowicz, G. Bjornsson, \& J. E. Pringle (Cambridge: Cambridge University Press), 148

Nelson, C. H., \& Whittle, M. 1995, ApJS, 99, 67

Nelson, C. H., \& Whittle, M. 1996, ApJ, 465, 96

Novikov, I. D., \& Throne, K. 1973, in Black Holes, ed. C. DeWitt, \& B. DeWitt (New York: Gordon and Breach), 343

Osterbrock, D. E., \& Martel, A. 1993, ApJ, 414, 552

Rees, M. J. 1984, ARA\&A, 22, 471

Sadler, E. M., Jenkins, C. R., \& Kotanyi, C. G. 1989, MNRAS, 240,591

Sadler, E. M., Slee, O. B., Reynolds, J. E., \& Roy, A. L. 1995, MNRAS, 276, 1373

Schmidt, M., \& Green, R. F. 1983, ApJ, 269, 352

Shakura, N. I., \& Sunyaev, R. A. 1973, A\&A, 24, 337

Silk, J., \& Rees, M. 1998, A\&A, 331, 1

Simien, F., \& de Vaucouleurs, G. 1986, ApJ, 302, 564

Véron-Cetty., M. P., \& Véron, P. 2000, ESO Scient. Rep., 19, 1

Wandel, A. 1999, ApJ, 519, L39

Wandel, A., Peterson, B. M., \& Malkan, M. A. 1999, ApJ, 526, 579

Wang, Y. P., Biermann, P. L., \& Wandel, A. 2000, A\&A, 361, 550

Whittle, M. 1992, ApJS, 79, 49

Worthey, G. 1994, ApJS, 95, 107 